\title{
DETC2013-13023
}

\section{VALIDATION OF THE INVERSE DYNAMIC ANALYSIS OF HUMAN GAIT USING A FORWARD DYNAMICS APPROACH}

\author{
Rosa Pàmies-Vilà \\ Department of Mechanical Engineering and \\ Biomedical Engineering Research Centre, \\ Universitat Politècnica de Catalunya, \\ Barcelona, Catalunya, Spain \\ rosa.pamies@upc.edu
}

\author{
Josep M. Font-Llagunes \\ Department of Mechanical Engineering and \\ Biomedical Engineering Research Centre, \\ Universitat Politècnica de Catalunya, \\ Barcelona, Catalunya, Spain \\ josep.m.font@upc.edu
}

\begin{abstract}
One of the aims of the dynamic analysis of human gait is to know the joint forces and torques that the musculoskeletal system produces during the motion. For this purpose, an 18 segment $3 D$ model with 57 degrees of freedom is implemented. The analysis of a captured motion can be addressed by means of forward or inverse dynamic analyses. In this work, both analyses are computed using multibody dynamics techniques. The forward dynamic analysis is carried out with the aim of simulating the movement of the multibody system using the results of the inverse problem as input data. Since the inverse analysis is solved using a dynamically consistent methodology, the forward dynamic analysis allows us to simulate up to the $90 \%$ of the gait cycle without any controller. After that, a proportional derivative (PD) controller is implemented to stabilize the system, which gets to simulate the complete captured motion. Moreover, the dynamic contribution of the controller is really low and the simulated motion is extremely close to the original one. The methodology presented allows us to validate the correctness of the inverse dynamics analysis and it is an intermediate step towards the prediction problem: it requires dynamical consistency too, but the uncertainties involved in the problem are lower than in a predictive approach.
\end{abstract}

\section{NOMENCLATURE}

$\begin{array}{ll}\mathbf{e}(t) & \text { error signal in the PD control scheme } \\ \mathbf{q}=\left\{q_{1}, \ldots, q_{n}\right\}^{T} & \text { vector of generalized coordinates } \\ \dot{\mathbf{q}} & \text { vector of generalized velocities } \\ \ddot{\mathbf{q}} & \text { vector of generalized accelerations }\end{array}$

\begin{tabular}{|c|c|}
\hline$\{\mathbf{u}, \mathbf{v}, \mathbf{w}\}_{S}$ & $\begin{array}{l}\text { unit vectors used to define the local basis of } \\
\text { segment } S\end{array}$ \\
\hline $\mathbf{z}$ & vector of independent coordinates \\
\hline$\dot{\mathbf{z}}$ & vector of independent velocities \\
\hline$\ddot{\mathbf{z}}$ & vector of independent accelerations \\
\hline $\mathbf{I}_{i}$ & identity matrix $(i \times i)$ \\
\hline$K_{d}$ & derivative coefficient of the PD controller \\
\hline$K_{p}$ & proportional coefficient of the PD controller \\
\hline$K_{p}^{i}$ & proportional gain associated with segment $i$ \\
\hline $\mathbf{M}$ & global system mass matrix \\
\hline$T$ & kinetic energy \\
\hline$(\alpha, \beta, \gamma)_{s}$ & orientation angles of segment $S$ \\
\hline$\lambda$ & vector of Lagrange multipliers \\
\hline$\Phi$ & vector of kinematic constraints \\
\hline$\Phi_{\mathrm{q}}$ & Jacobian matrix of the kinematic constraints \\
\hline$\dot{\boldsymbol{\Phi}}_{\mathrm{q}}$ & time derivative of $\boldsymbol{\Phi}_{\mathbf{q}}$ \\
\hline$\Phi_{t}$ & $\begin{array}{l}\text { vector containing the partial derivatives of } \\
\text { the constraints with respect to time }\end{array}$ \\
\hline$\dot{\boldsymbol{\Phi}}_{t}$ & time derivative of $\boldsymbol{\Phi}_{t}$ \\
\hline
\end{tabular}

\section{INTRODUCTION}

The human body can be modeled as a set of bones linked by joints forming the skeleton, and a set of soft tissues (flesh, organs and muscles). Using multibody dynamic techniques, multi-segment models can be created with relative ease and low 
computational cost. The motion between the segments can be described by kinematic restrictions and the classical mechanical concepts provide us with the possibility of estimating the dynamic variables of the motion.

One of the aims of human motion dynamic analysis is to know the forces and torques developed by the musculoskeletal system during a movement. Multibody dynamics techniques can provide quantitative results for a three dimensional motion using non-invasive devices and with minimal interference with the subject's motion. Depending on the purpose of the study, multibody dynamics techniques can be employed in two different ways: inverse and forward dynamics [1].

Inverse Dynamic Analysis (IDA) is used to calculate the net joint reaction forces and driver torques that the musculoskeletal system produces during human locomotion using acquired kinematic and kinetic data and estimated body segment parameters (BSP). This can be helpful for recognizing normal and pathological gait patterns. Conversely, Forward Dynamic Analysis (FDA) is used to predict the body movement from known muscle forces (or resultant joint torques) using principles of neural science (neurocontrol) or optimal control. This approach can be adequate for investigating aspects of muscle function and energetic cost, for simulating gait disorders or for predicting the combined actuation of the musculoskeletal system and assistive devices, such as exoskeletons or orthoses.

The forward dynamics approach requires a control strategy for the actuation, and the resulting motion is computed through integration of the equations of motion. In recent years, new methods for efficient control of the musculoskeletal system using optimal control methods have been presented [2-5]. A growing interest in motion prediction has appeared during the last years, e.g., to anticipate the result of surgery, to help in the design of prosthetic/orthotic devices, or to study human motion dynamics performing various tasks [6, 7]. To this end, the motion parameters can be considered as design variables of an optimization problem. In this context, the determination of the joint efforts for a given motion (inverse dynamic analysis) is a required step for the subsequent evaluation of cost functions and constraints.

In this work, both inverse and forward dynamics approaches are considered. Regarding the IDA, the movement of the subject is recorded in the laboratory using a motion capture system that provides the position along time of 37 markers attached on the body. Position data are filtered using an algorithm based on Singular Spectrum Analysis (SSA) and the natural coordinates of the model are calculated using algebraic relations among the marker positions. Afterwards, a procedure ensures the kinematic consistency and the data processing continues with the approximation of the position histories using B-spline curves. The velocity and acceleration values are then obtained by analytical derivation. Once the data are processed, the IDA is computed and the joint torques and foot-ground contact wrenches are calculated. The double support indeterminacy is solved using the Corrected Force Plate (CFP) sharing approach developed by the group [8]. This method allows combining the force plate data and the markers information to obtain a set of dynamically consistent data, with no need either to modify the motion or to add residual wrenches. Regarding the FDA, in a first step it is carried out without any control strategy allowing us to simulate up to the $90 \%$ of the gait cycle, when a divergence from the expected motion started. For this reason, and in order to increase the percentage of the gait cycle simulated, a PD controller is implemented.

Although the IDA and the FDA approaches are separately common research topics in the field of biomechanics, a small number of studies prove the validity of the obtained results. The comparison between the results of a specific study and the ones present in the literature could not be enough for the validation of the results. Biomechanical analyses present a high amount of uncertainties and the results usually are plotted as the average value bounded by the standard deviation (which is large). Being within the limits does not guarantee the correctness of those results.

In this work, the results of the IDA are used as input data for the FDA; and the results of the latter (the motion) have been compared with the motion capture in the laboratory (input of the IDA). In an ideal case, the motion obtained through the forward simulation should be the original captured motion. : as long as the inverse and forward models are the same, the results would be close. However, the joint torques published in the literature (as a result of an IDA) are not always are dynamically consistent with the captured motion. And thus, if they are used to compute an FDA, the original motion is not obtained.

Usually, in a biomechanics laboratory, the motion is recorded using a motion capture system and the contact interaction between the foot and the ground is measured by means of force plates. These devices provide the individual foot-ground contact wrench along the gait. If the kinematic information of the whole-body is known and the results from two force plates are used as inputs of the IDA (employing one plate for each foot-ground contact), the biomechanical system is overdetermined. Depending on the method used to solve this problem, the IDA results could not be dynamically consistent with the motion.

For example, in order to obtain a single unique solution during the double support phase, some studies add a set of force and torque components (the residual wrench) to a segment (usually pelvis or trunk). The residual wrench does not have physical meaning and, in an ideal case, this wrench should be null. However, due to the errors in the data, it is not zero in general and some studies try to minimize this wrench using optimization techniques and modifying the BSP [9], the joint trajectories [10] or the joint accelerations [11-12]. In those cases, the final joint torques are not dynamically consistent with the captured motion. Thus, it is not possible to use inverse dynamics results as inputs of forward simulation without 
including these nonphysical residual wrenches. Moreover, all these studies assume that the residual torques are small, and this assumption highly depends on the accuracy of the input measurements.

Another method to solve the double support indeterminacy is to perform a least-squares inverse dynamics approach [13]. In this study, the constraints formed by imperfect measurements are relaxed and a static optimization problem is used to adjust the angular acceleration of the segments and the foot-ground contact forces to obtain the solution that is simultaneously most consistent with measured accelerations and contact forces. However, the estimates of the torques are not dynamically consistent over time and thus, the resulting accelerations, when integrated in a forward dynamic analysis, would not reproduce the original motion.

In both cases, if a FDA is computed using the IDA result as input data, the obtained motion will not match the original one. Since the IDA carried out in this work gives a set of dynamically consistent forces and torques, without adding residual wrenches nor modifying the motion, the use of the FDA allows us to validate the accuracy of the inverse dynamics methodology used and it represents a first step towards human gait prediction.

\section{DYNAMICS MODELING}

\section{Biomechanical Model}

The human body is modeled as a multibody system formed by rigid bodies, an approach which has been widely used to analyze human gait $[1,14-16]$. The modeling of the human body by means of rigid bodies is a reasonable assumption if the motion involves big movements without strong impacts as in human locomotion [14].

The 3D model used consists of 18 anatomical segments: two hindfeet, two forefeet, two shanks, two thighs, pelvis, torso, neck, head, two arms, two forearms and two hands. The segments are linked by ideal spherical joints defining a 57 degree of freedom (DOF) model.

The kinematic information of the motion is obtained from the trajectories of a set of 37 markers attached to the human body (Figure 1(a)). The marker positions are used to determine the orientation of each rigid body and to define the position of the 17 anatomical joints (Figure 1(b)). The horizontal global axis $\mathrm{X}$ points to the direction of motion, the $\mathrm{Z}$ axis is perpendicular to the floor and pointing upwards, and the $Y$ axis is defined so that $\{\mathrm{X}, \mathrm{Y}, \mathrm{Z}\}$ is a positive defined orthonormal basis.

The configuration of a body segment is defined through the position of one of its points and its orientation with respect to the ground frame. For all segments, the chosen point will be

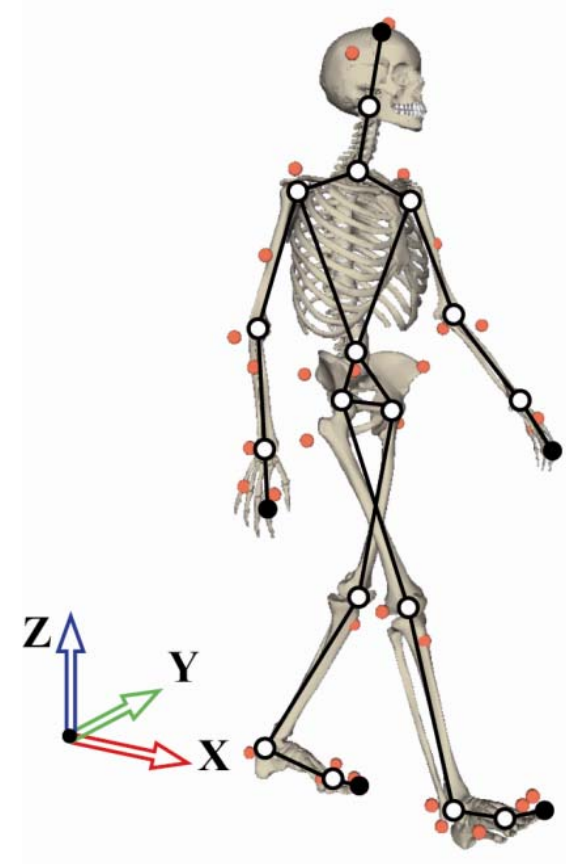

(a)

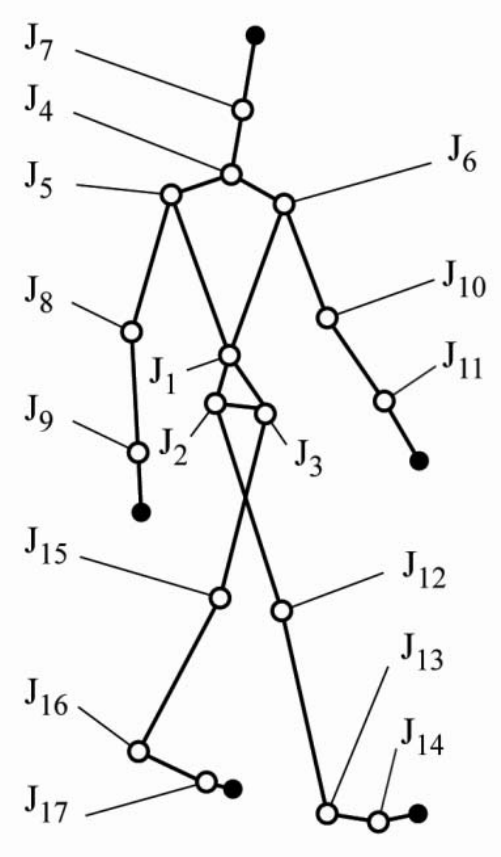

(b)

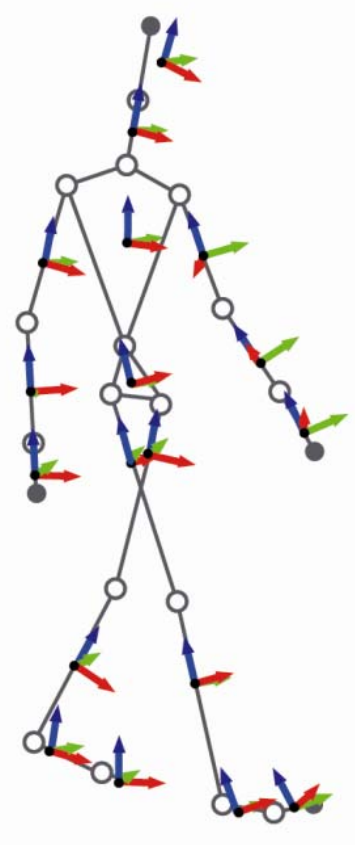

(c)

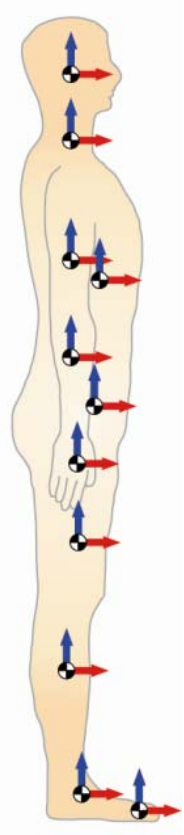

(d)

Figure 1. Biomechanical model used: (a) 3D model of the human body. (b) Numeration of the seventeen joints. (c) Points and unit vectors defining the model in a general posture. (d) Sagittal view of the model at the reference posture. 
the proximal joint. In order to compute the orientation, local bases $\left\{\mathrm{X}^{\prime}, \mathrm{Y}^{\prime}, \mathrm{Z}^{\prime}\right\}_{s}$ are defined for all segments (Figure 1(c)). Unit vectors $\{\mathbf{u}, \mathbf{v}, \mathbf{w}\}_{S}$ are used to define the axes of the local bases. Note that in the reference posture, the orientation of each local basis matches the one of the absolute basis $\{\mathrm{X}, \mathrm{Y}, \mathrm{Z}\}$ (Figure 1(d)).

The 3D model of the subject has been developed in mixed (natural plus angular) coordinates and it is modeled using 228 dependent coordinates: 22 points (which correspond to the positions of all the spherical joints, along with the centers of mass of the five extreme segments: head, hands and forefeet) plus 2 orthogonal unit vectors for each rigid body (so, 36 vectors). Both, points and vectors are expressed using three Cartesian coordinates, thus making a total of 174 variables. The remaining 54 variables are the 18 sets of 3 angles that define the orientation of each segment with respect to the absolute frame.

\section{Gait Analysis Laboratory}

The subject selected to perform the experiments is a healthy adult male, 34 years old, mass $85 \mathrm{~kg}$ and height $1,82 \mathrm{~m}$. He walks on a walkway that encloses two force plates (AMTI, AccuGait sampling at $100 \mathrm{~Hz}$ ). The motion is captured by 12 optical cameras (Natural Point, OptiTrack FLEX:V100 also sampling at $100 \mathrm{~Hz}$ ) that compute the position of 37 optical markers. An SSA procedure is applied to these position histories in order to reduce the noise due to the motion capture process.

The process used to guarantee kinematic data consistency at position level is explicitly described in [17]. From the kinematically consistent data set obtained above, a set of independent coordinates $\mathbf{z}$ is calculated: the three Cartesian coordinates of the proximal pelvis joint, and the three angles of each segment with respect to the global frame, defining a total of 57 degrees of freedom (DOF).

Prior to differentiating these configuration histories, the SSA filter is applied again to reduce the noise introduced by the kinematic consistency processing. Note that since the chosen independent coordinates define the position of one point and the orientation of each segment, these new histories are also kinematically consistent.

The sample rate of the data collected at the laboratory $(100 \mathrm{~Hz})$ is not enough to compute forward dynamics, thus, a smoother interpolation is required. For this purpose, B-spline functions are used. Finally, differentiating these curves, the velocity and acceleration of the independent coordinates, $\dot{\mathbf{z}}$ and $\ddot{\mathbf{z}}$ are determined.

\section{Multibody Formulation}

There are several procedures to obtain the dynamic equations of motion. In this work, the analysis is formulated using a multibody dynamics methodology. The equations of motion can be expressed using dependent coordinates as:

$$
\frac{d}{d t}\left(\frac{\partial T}{\partial \dot{\mathbf{q}}}\right)-\frac{\partial T}{\partial \mathbf{q}}+\boldsymbol{\Phi}_{\mathbf{q}}^{T} \boldsymbol{\lambda}=\mathbf{Q}
$$

where $T$ is the kinetic energy of the multibody system, $\mathbf{Q}$ is the vector of generalized forces and $-\boldsymbol{\Phi}_{\mathrm{q}}^{T} \boldsymbol{\lambda}$ are the generalized forces associated to the Lagrange multipliers $\lambda$. The kinetic energy can be written as:

$$
T=\frac{1}{2} \dot{\mathbf{q}}^{T} \mathbf{M} \dot{\mathbf{q}}
$$

where $\mathbf{M}$ is the mass matrix of the system. Note that using the proposed natural coordinates this matrix is constant. Therefore, Eq. (1) can be written as:

$$
\mathbf{M} \ddot{\mathbf{q}}+\boldsymbol{\Phi}_{\mathbf{q}}^{T} \boldsymbol{\lambda}=\mathbf{Q}
$$

The system of equations in Eq. (3) contains $n$ equations, where $n$ is the total number of coordinates. The number of unknown variables is $n+m$, where $m$ is the number of constraints which coincides in turn with the $m$ Lagrange multipliers in $\lambda$. Therefore, the system can be written as:

$$
\left\{\begin{array}{c}
\mathbf{M} \ddot{\mathbf{q}}+\mathbf{\Phi}_{\mathbf{q}}^{T} \lambda=\mathbf{Q} \\
\mathbf{\Phi}(\mathbf{q}, t)=\mathbf{0}
\end{array}\right.
$$

Eq. (4) represents a Differential Algebraic Equation (DAE) system with $n$ differential equations and $m$ algebraic equations. The strategy to solve this DAE system is based on turning it into an Ordinary Differential Equation (ODE) system, since there are many well-known methods for its integration. In this work, to solve the motion equations a velocity projection method proposed in [18] is used. The equations are reduced to their state-space form, i.e., their independent coordinates $\dot{\mathbf{z}}$. The time derivative of the constraint equations yields:

$$
\dot{\boldsymbol{\Phi}}(\mathbf{q}, \dot{\mathbf{q}}, t)=\boldsymbol{\Phi}_{\mathbf{q}} \dot{\mathbf{q}}+\boldsymbol{\Phi}_{t}=0 ; \quad \boldsymbol{\Phi}_{t} \equiv-\mathbf{b}
$$

The independent velocities $\dot{\mathbf{z}}$ are chosen from the dependent ones $\dot{\mathbf{q}}$ and a matrix $\mathbf{B}$ can be defined, such that:

$$
\dot{\mathbf{z}}=\mathbf{B} \dot{\mathbf{q}}
$$

Then, using Eqs. (5) and (6), it is obtained that:

$$
\left[\begin{array}{c}
\boldsymbol{\Phi}_{\mathbf{q}} \\
\mathbf{B}
\end{array}\right] \dot{\mathbf{q}}=\left[\begin{array}{cc}
\boldsymbol{\Phi}_{\mathbf{q}}^{\mathrm{d}} & \boldsymbol{\Phi}_{\mathbf{q}}^{\mathrm{i}} \\
\mathbf{0}_{g \times m} & \mathbf{I}_{g}
\end{array}\right]\left\{\begin{array}{c}
\dot{\mathbf{q}}^{\mathrm{d}} \\
\dot{\mathbf{q}}^{\mathrm{i}}
\end{array}\right\} \equiv\left\{\begin{array}{c}
\mathbf{b} \\
\dot{\mathbf{z}}
\end{array}\right\}
$$

where superscripts "d" and "i" indicate dependent and independent coordinates respectively, $g=n-m$ is the number of degrees of freedom and $\mathbf{I}_{g}$ is the $g \times g$ identity matrix. From Eq. (7), it can be deduced that the rows of matrices $\boldsymbol{\Phi}_{\mathbf{q}}$ and $\mathbf{B}$ are linearly independent. Therefore, vector $\dot{\mathbf{q}}$ can be calculated inverting the matrix of the $n \times n$ linear system: 


$$
\dot{\mathbf{q}}=\left[\begin{array}{c}
\boldsymbol{\Phi}_{\mathbf{q}} \\
\mathbf{B}
\end{array}\right]^{-1}\left\{\begin{array}{l}
\mathbf{b} \\
\dot{\mathbf{z}}
\end{array}\right\} \equiv \mathbf{S b}+\mathbf{R} \dot{\mathbf{z}}
$$

and identifying terms [19]:

$$
\begin{aligned}
& \mathbf{S}=\left[\begin{array}{l}
\mathbf{S}^{\mathrm{d}} \\
\mathbf{S}^{\mathrm{i}}
\end{array}\right]=\left[\begin{array}{c}
\mathbf{\Phi}_{\mathrm{q}}^{\mathrm{d}} \\
\mathbf{0}_{g \times m}^{-1}
\end{array}\right] \\
& \mathbf{R}=\left[\begin{array}{l}
\mathbf{R}^{\mathrm{d}} \\
\mathbf{R}^{\mathrm{i}}
\end{array}\right]=\left[\begin{array}{c}
-\left[\begin{array}{c}
\boldsymbol{\Phi}_{\mathbf{q}}^{\mathrm{d}} \\
\mathbf{I}_{g}^{-1}
\end{array}\right] \boldsymbol{\Phi}_{\mathbf{q}}^{\mathrm{i}} \\
\mathbf{I}_{g}
\end{array}\right]
\end{aligned}
$$

The acceleration equation can be similarly determined:

$$
\ddot{\boldsymbol{\Phi}}(\mathbf{q}, \dot{\mathbf{q}}, \ddot{\mathbf{q}}, t)=\boldsymbol{\Phi}_{\mathbf{q}} \ddot{\mathbf{q}}+\dot{\boldsymbol{\Phi}}_{\mathbf{q}} \dot{\mathbf{q}}+\dot{\boldsymbol{\Phi}}_{t}=\mathbf{0} ; \quad \boldsymbol{\Phi}_{\mathbf{q}} \ddot{\mathbf{q}}=-\dot{\boldsymbol{\Phi}}_{t}-\dot{\boldsymbol{\Phi}}_{\mathbf{q}} \dot{\mathbf{q}} \equiv \mathbf{c}
$$

Therefore, since $\mathbf{B}$ is constant:

$$
\begin{gathered}
{\left[\begin{array}{c}
\Phi_{\mathbf{q}} \\
\mathbf{B}
\end{array}\right] \ddot{\mathbf{q}} \equiv\left\{\begin{array}{l}
\mathbf{c} \\
\ddot{z}
\end{array}\right\}} \\
\ddot{\mathbf{q}}=\left[\begin{array}{c}
\boldsymbol{\Phi}_{\mathbf{q}} \\
\mathbf{B}
\end{array}\right]^{-1}\left\{\begin{array}{l}
\mathbf{c} \\
\ddot{\mathbf{z}}
\end{array}\right\}=\mathbf{S c}+\mathbf{R} \ddot{\mathbf{z}}
\end{gathered}
$$

If an IDA is performed using this formulation, the only unknown variable in Eq. (4) is the vector of Lagrange multipliers $\lambda$, which can be perfectly determined at each time instant. In an FDA, motion is not known and Eq. (4) presents fewer equations than unknowns, it is an underdetermined system. As a result, to determine the motion it is necessary to establish the dynamic equilibrium condition that leads to a second order differential system. The equations of motion can be written as:

$$
\begin{gathered}
\mathbf{R}^{T} \mathbf{M R} \ddot{\mathbf{z}}=\mathbf{R}^{T}(\mathbf{Q}-\mathbf{M}(\dot{\mathbf{R}} \dot{\mathbf{z}}+\mathbf{S} \dot{\mathbf{b}}+\dot{\mathbf{S}} \mathbf{b})) \\
\mathbf{S c}=\dot{\mathbf{R}} \dot{\mathbf{z}}+\mathbf{S} \dot{\mathbf{b}}+\dot{\mathbf{S}} \mathbf{b}
\end{gathered}
$$

and, therefore:

$$
\mathbf{R}^{T} \mathbf{M R} \ddot{\mathbf{z}}=\mathbf{R}^{T}(\mathbf{Q}-\mathbf{M S c})
$$

which can be expressed in a compact form as:

$$
\tilde{\mathbf{M}} \ddot{\mathbf{z}}=\tilde{\mathbf{Q}}
$$

Eq. (17) is a system of $g=n-m$ equations with $g$ unknowns, which can be easily solved for the independent accelerations $\ddot{\mathbf{z}}$. Vectors $\mathbf{z}$ and $\dot{\mathbf{z}}$ are obtained by numerical integration. There are several numerical algorithms in the literature to integrate the equations of motion when they have been transformed into a second order ODE system [18]. In this work, the well-known trapezoidal rule is used. After that and using Eqs. (8) and (13), the dependent variables $\mathbf{q}, \dot{\mathbf{q}}$ and $\ddot{\mathbf{q}}$ can be calculated. Finally, the Lagrange multipliers (which are related to the joint wrenches) can be obtained from Eq. (4).

\section{RESULTS AND DISCUSSION}

\section{Inverse Dynamics Results}

Using the kinematic information of the whole-body and combining the equations of motion of all the segments, the total contact forces acting on the system and the torques between the ground and each segment can be calculated. Since, during walking, the only unknown external forces and torques acting on the human body are the foot-ground contact forces, the global external wrench can be determined. This total wrench corresponds to the actual foot-ground contact wrench during the single support phase. However, during the double support phase, how this wrench is distributed between the two feet cannot be known from kinematic information only. This is known as the double support sharing problem or indeterminacy.

In order to compute the IDA, the double support indeterminacy needs to be solved, that is, how the kinetic and kinematic information from the laboratory are combined to achieve a univocal dynamic solution. To this end, different techniques have been proposed in the literature [15, 13, 20-24]. Generally, this indeterminacy is overcome by the measurement of the individual foot-ground contact wrenches by means of force plates [22-24]. However, if two force plate measurements are used together with the whole-body kinematic information (traditional approach), as said before, the inverse dynamic problem becomes overdetermined.

In this work, in order to preserve the kinematics, the Corrected Force Plate (CFP) sharing approach developed by the group is applied [13, 20]. This method allows combining the force plate data and the markers information to obtain a set of dynamically consistent data, with no need either to modify the motion or to add residual wrenches. Moreover, the joint torques obtained via the IDA have physical meaning and can be used to compute the FDA.

After solving the double support sharing problem using the CFP method, all the joint torques are calculated. It has been shown that the obtained results present a good correlation when compared with other results already published in the literature [25]. Figure 2 shows the net torques in the lower limb joints (sagittal plane). The dashed line and the grey area correspond respectively to the average torque bounded by the standard deviation obtained in [25]. The recorded motion contains more than one cycle. It covers all instants with force plate information available. It starts at the heel strike of the right foot ( $0 \%$ of gait cycle), includes also the next heel strike of the same foot (100\%) and finishes at the toe off of the left foot belonging to the next cycle ( 116 \%). 
Since the methodology applied in [25] uses force plate data as input information to compute the IDA, and only two force plate devices were available in the lab, the results provide information of only one double support of the gait cycle, when both feet contact force plates (Note that [25] provides the results of the $100 \%$ of a gait cycle separately for each leg,

Right leg

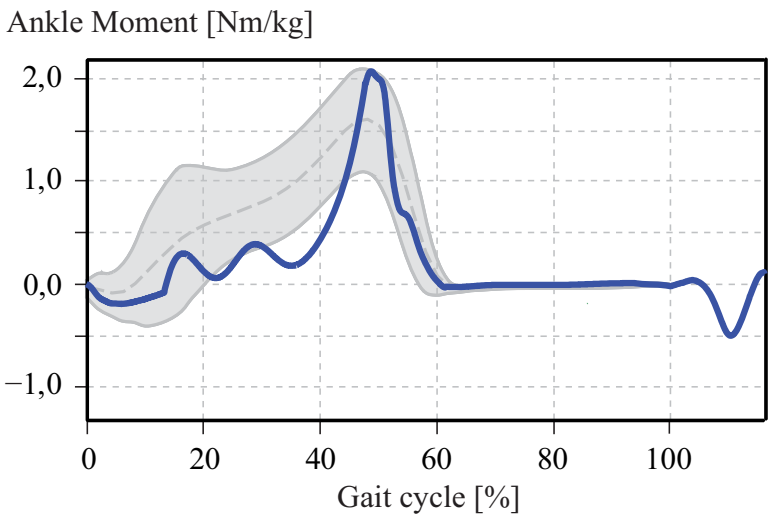

Knee Moment $[\mathrm{Nm} / \mathrm{kg}]$

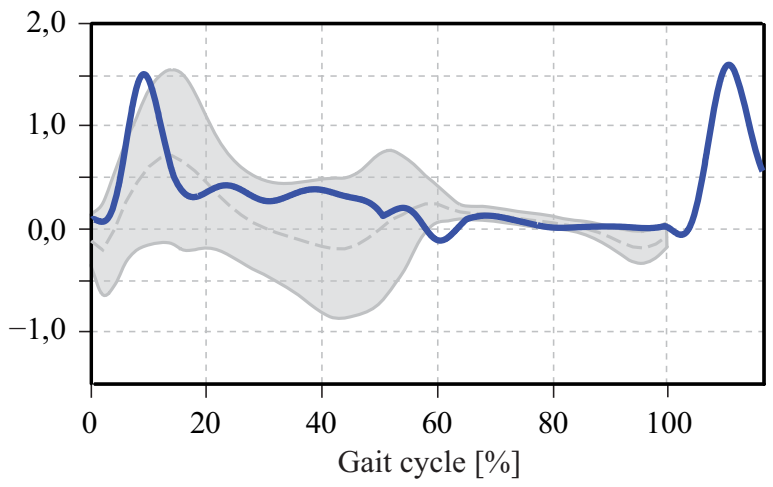

Hip Moment $[\mathrm{Nm} / \mathrm{kg}]$

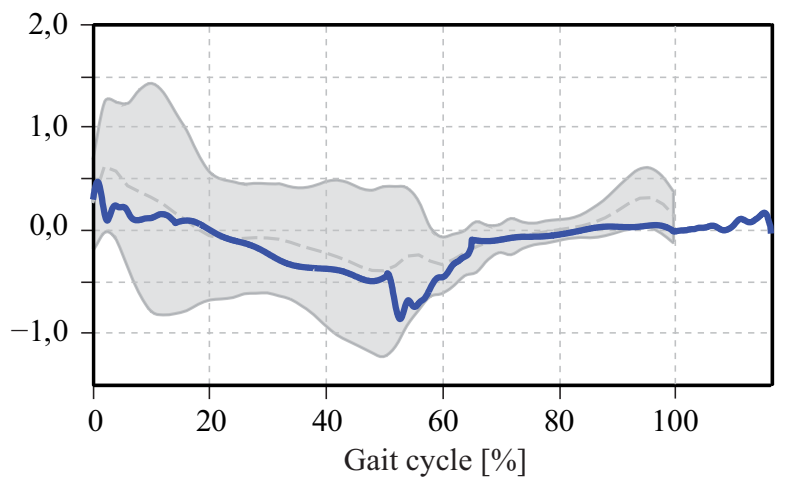

therefore two double support phases. However, only one double support is common for both legs). In contrast, as the CFP method can be applied using only one force plate, Figure 2 shows $\sim 116 \%$ of a cycle including three complete double support phases.
Left leg

Ankle Moment $[\mathrm{Nm} / \mathrm{kg}]$

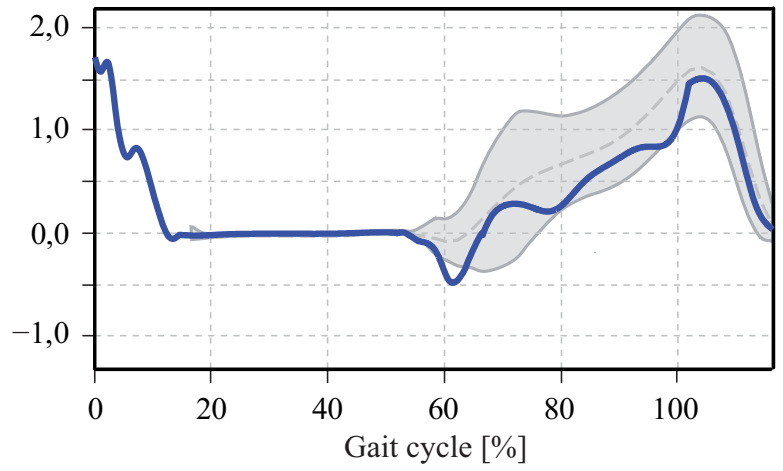

Knee Moment [ $\mathrm{Nm} / \mathrm{kg}$ ]

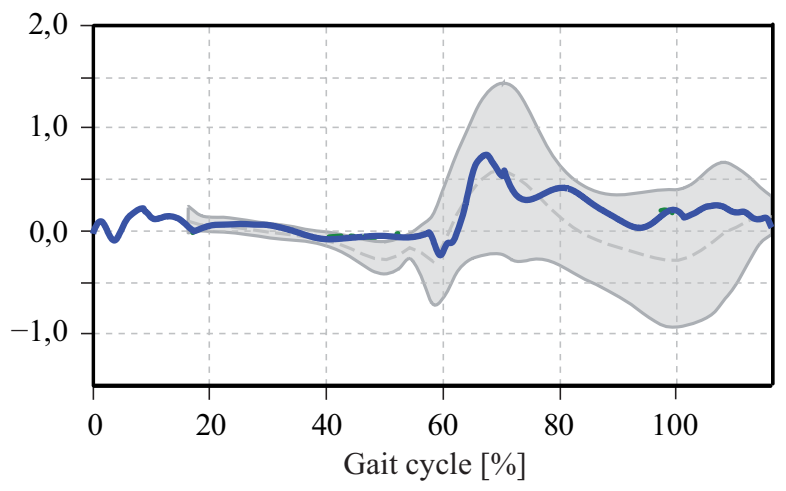

Hip Moment $[\mathrm{Nm} / \mathrm{kg}]$

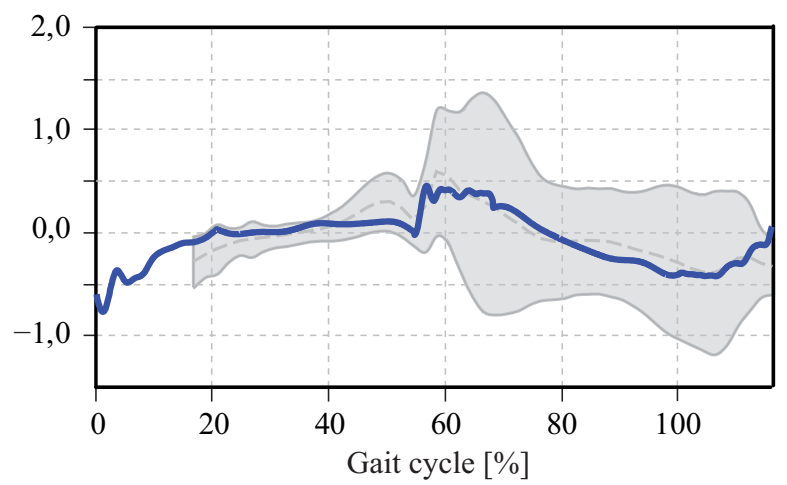

Winter average moment \pm Std. Dev. Inverse Dynamics Results using CFP

Figure 2. Ankle, knee and hip joint torques calculated using the CFP sharing method and comparison with Winter's results [25]. 


\section{Forward Dynamics Results}

The obtained dynamic information can be used as input of an FDA to simulate the dynamic response of the human body to this particular set of applied forces and torques. In this case, the principal objective of the analysis is to predict the movement of the multibody system. In an ideal case, the motion obtained through FDA using the results of the IDA as inputs should be the original motion captured at the laboratory (since our IDA results are dynamically consistent). However, in a real application, the simulated motion differs from the captured one due to errors in the integrator and stability and numerical problems

In a first attempt, the FDA was computed using a time step $\Delta \mathrm{t}=0,01 \mathrm{~s}$. However, using this interval, the multibody system became unstable and it was not possible to simulate the entire motion. At this point, the IDA needs to be recalculated using a smaller time step. The use of spline functions to define the motion allows obtaining kinematic information at any instant.

\section{Right leg}

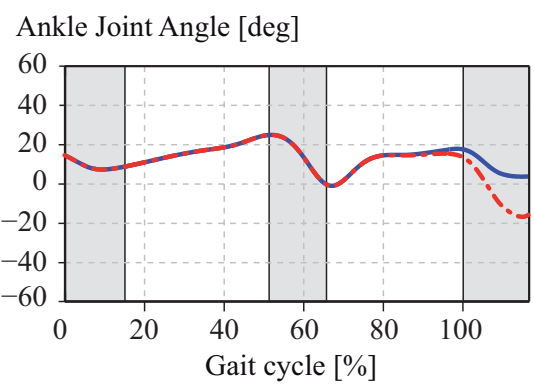

Knee Joint Angle [deg]

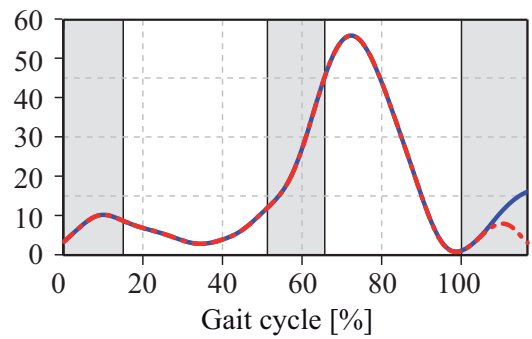

Hip Joint Angle [deg]

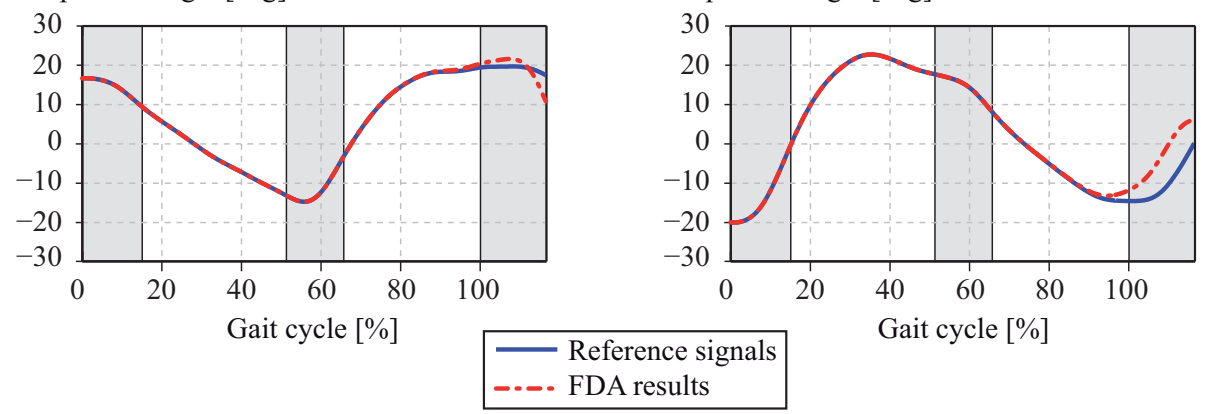

Figure 3. Ankle, knee and hip flexion angles (sagittal plane). Reference signals vs. FDA results.

\footnotetext{
Figure 3. Ankle, knee and hip flexion angles (sagittal plane). Reference signals vs. FDA results.
}

Therefore, a time step $\Delta \mathrm{t}=0,001 \mathrm{~s}$ is chosen and the IDA results are again recalculated.

The external forces and torques (calculated at $\Delta t=0,001 \mathrm{~s}$ ) are used as inputs of the FDA and the resultant motion is obtained. Figure 3 shows the joint flexion-extension angles (in red) and their actual values (in blue) for the ankle, knee and hip joints of both legs. It can be observed that during about the $90 \%$ of the cycle both curves overlap; the two motions are similar. However, at the end of the simulation, both curves begin to differ and the obtained motion does not correspond to normal human gait. Note that using the proposed inverse dynamics method; the obtained dynamic results do not contain residual torques. The FDA is computed using only external torques and forces, without any controller and, still, the results shown in Figure 3 are accurate. However, it can be noted that at the end of the simulation, the obtained motion differs from the original one due to integration errors, and stability and numerical problems.

\section{Left leg}

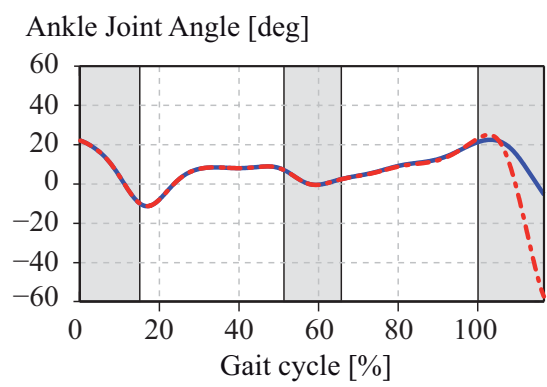

Knee Joint Angle [deg]

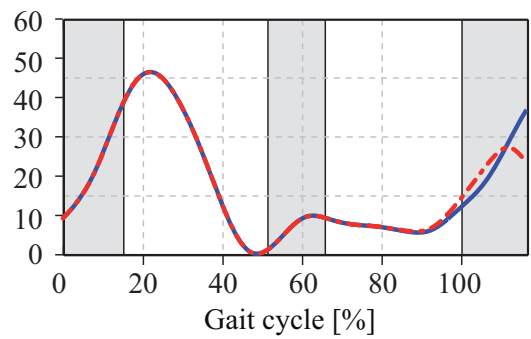

Hip Joint Angle [deg] 
In order to improve these results for the whole cycle, a proportional derivative (PD) controller is implemented. The control unit presents a typical structure of an elementary closed-loop system [26]. The controller is implemented in discrete time form, and the reference signals (the path that the system must follow) are the original time evolution of the degrees of freedom $\mathbf{z}^{\text {ref }}$, that is, the position of the lumbar joint and the three angles defining the absolute orientation of each segment.

The error and the error time derivative have the following expressions at each instant $t_{k}(k=1, \ldots, N$, where $N$ is the number of time steps of the simulation):

$$
\begin{gathered}
\mathbf{e}\left(t_{k}\right)=\mathbf{z}^{r e f}\left(t_{k}\right)-\mathbf{z}\left(t_{k}\right) ; \quad \mathbf{e}\left(t_{0}\right)=0 \\
\frac{d \mathbf{e}\left(t_{k}\right)}{d t}=\frac{\mathbf{e}\left(t_{k}\right)-\mathbf{e}\left(t_{k-1}\right)}{\Delta t}
\end{gathered}
$$

The controller is implemented and the respective actuators are adopted. The actuators have the function of converting the position and angular error differences into the corresponding generalized forces. There are 3 linear actuators and 54 angular ones. Therefore, the controller outputs are the external lumbar joint forces and the absolute segment torques that are needed to counteract the deviation of the controlled variable $\mathbf{z}$ from the prescribed reference $\mathbf{z}^{\text {ref }}$.

The proportional gain $K_{p}^{i}$ associated with segment $i$ is proportional to the mass of the segment $m_{i}$ :

$$
K_{p}^{i}=\tilde{K}_{p} m_{i}
$$

where $\tilde{K}_{p}=400([\mathrm{~N} / \mathrm{kg} \mathrm{m}]$ or $[\mathrm{Nm} / \mathrm{kg} \mathrm{rad}]$ depending on whether the actuator is linear or rotative, respectively). The contribution of the derivative term is proportional (by the value of $K_{p}^{i} T_{d}$ ) to the slope of the error over time, i.e., its first derivative with respect to time, Eq. (19). In our case, $T_{d}=2,5 \cdot 10^{-5} \mathrm{~s}$. The values of $\tilde{K}_{p}$ and $T_{d}$ have been chosen in order to avoid oscillations and minimize overshoot. Note that these values are adjusted by trial-and-error work.

Once this procedure is implemented, it can be observed that the biomechanical model is able to follow the prescribed

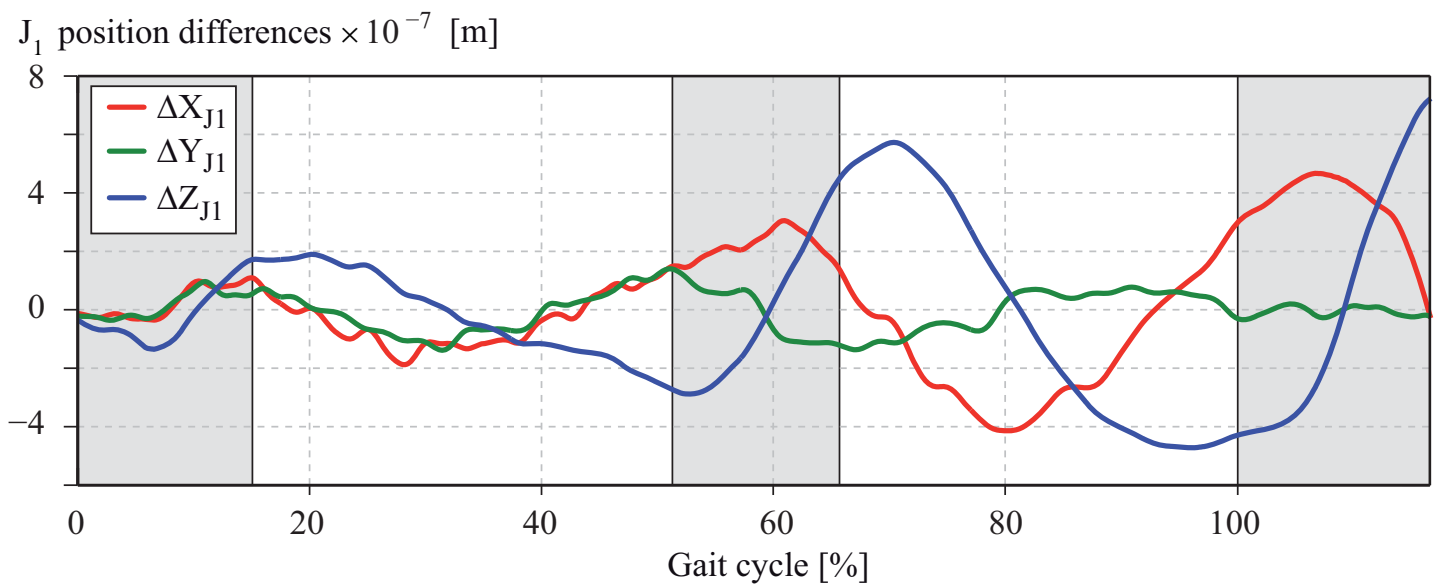

Right leg angular differences $\times 10^{-6}[\mathrm{rad}]$

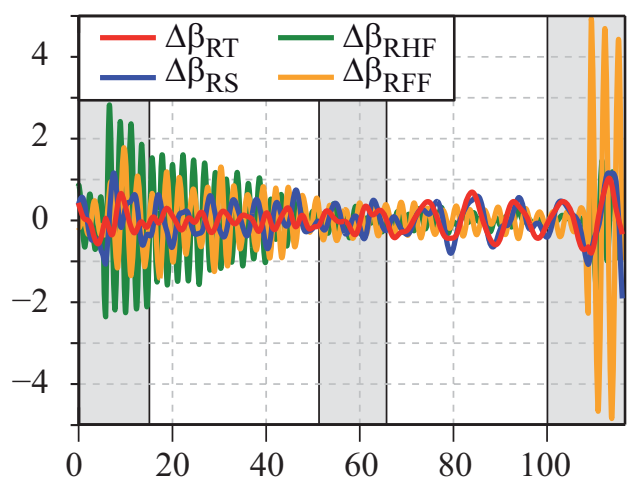

Gait cycle [\%]

(b) (a)

Left leg angular differences $\times 10^{-6}$ [rad]

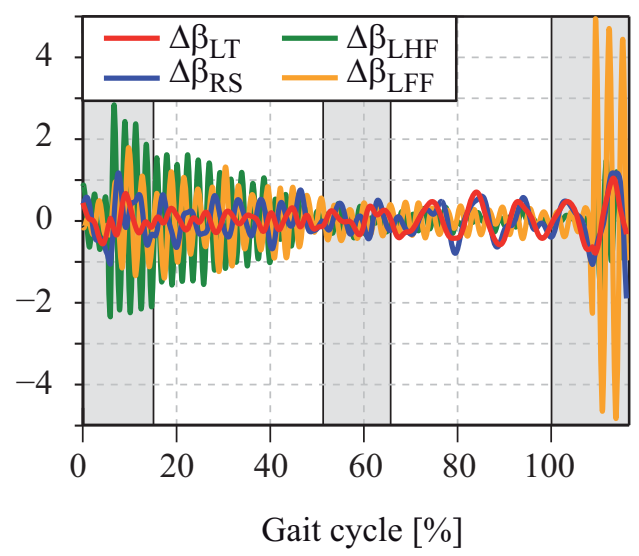

(c)

Figure 4. Differences between the reference signal and the signal obtained through FDA using the PD controller: (a) Position differences at lumbar joint. (b) and (c) Absolute angle $\beta$ differences for the thigh, shank, hindfoot and forefoot segments at right and left leg, respectively. 
motion. During all the analysis, the controlled variables are concurrent with the reference variables. Figure 4(a) shows the differences between the actual position of the lumbar joint $\mathrm{J}_{1}$ (captured motion) and the same variable calculated through forward dynamics. Similarly, Figures 4(b) and 4(c) show these differences for the angle $\beta$ (angle between each segment and the ground in Y direction) of the thigh, shank, hindfoot, and forefoot for both, right and left legs, respectively. Figure 4 depicts a small difference between the two signals $\left(<8 \cdot 10^{-7} \mathrm{~m}\right.$ in position and $<5 \cdot 10^{-6}$ rad in angle).

The biomechanical model followed the used reference, leading to the conclusion that this simple control scheme is enough to effectively control the biomechanical system during gait under a simulated condition. The dynamic effect of the controller is a force acting on the lumbar joint $\left(\mathrm{J}_{1}\right)$ and a set of torques acting on each segment.

As representative magnitudes, Table 1 shows the maximum values of the controller force $\left(\mathrm{F}_{\mathrm{X}, \mathrm{J1}}, \mathrm{F}_{\mathrm{Y}, \mathrm{J1}}, \mathrm{F}_{\mathrm{Z}, \mathrm{J1}}\right)$ and the maximum values of the absolute $\mathrm{Y}$ component of the torques for the thighs, shanks, hindfeet and forefeet of both legs $\left(\mathrm{M}_{\mathrm{RT}}\right.$, $\left.M_{R S}, M_{R H F}, M_{R F F}, M_{L T}, M_{L S}, M_{L H F}, M_{L F F}\right)$. The table also contains the corresponding instant of time when these peaks appear (expressed as a percentage of the gait cycle).

Table 1. Maximum dynamic contribution of the PD controller and its corresponding instant of time (in \% of gait cycle).

\begin{tabular}{lcc} 
& $\mathbf{F} \times \mathbf{1 0}^{-3}[\mathbf{N}]$ & Gait cycle [\%] \\
\hline $\mathrm{F}_{\mathrm{X}, 11}$ & 2,977 & 106,5 \\
$\mathrm{~F}_{\mathrm{Y}, 11}$ & 0,925 & 50,9 \\
$\mathrm{~F}_{\mathrm{Z}, 11}$ & 4,587 & 116,4 \\
\hline
\end{tabular}

\begin{tabular}{lcc} 
& $\left.\mathbf{M} \times \mathbf{1 0}^{-\mathbf{3}} \mathbf{[ N m}\right]$ & Gait cycle [\%] \\
\hline $\mathrm{M}_{\mathrm{RT}}$ & 3,808 & 113,6 \\
$\mathrm{M}_{\mathrm{RS}}$ & 1,762 & 113,5 \\
$\mathrm{M}_{\mathrm{RHF}}$ & 1,025 & 109,8 \\
$\mathrm{M}_{\mathrm{RFF}}$ & 0,556 & 114,4 \\
$\mathrm{M}_{\mathrm{LT}}$ & 2,586 & 114,4 \\
$\mathrm{M}_{\mathrm{LS}}$ & 1,795 & 112,2 \\
$\mathrm{M}_{\mathrm{LHF}}$ & 0,997 & 6,7 \\
$\mathrm{M}_{\mathrm{LFF}}$ & 0,440 & 109,8 \\
\hline
\end{tabular}

It can be seen that the dynamic contribution of the PD controller is less than $5 \cdot 10^{-3} \mathrm{~N}$ and $4 \cdot 10^{-3} \mathrm{Nm}$. These magnitudes are much lower than the range of variation of the joint forces and torques for a normal gait, and thus, the dynamic contribution of the PD controller is negligible. Note that all the peaks appear in double support instants (shadowed areas in Figure 3) and most of them at the end of the analyzed motion.
From the obtained results, it can be concluded that the joint efforts calculated using forward dynamics (input efforts plus controller dynamic contribution) and the ones calculated via an IDA are practically equivalent allowing us to validate the methodology used to compute the IDA using an FDA approach.

\section{CONCLUSIONS}

In this work, inverse and forward dynamic analyses have been implemented. The comparison between the inverse dynamics results and the literature ones is used as a first validation of the model. Moreover, the inverse dynamics results (obtained using the CFP sharing method) have been used as inputs of a forward dynamics problem, obtaining good results to simulate up to the $90 \%$ of the gait cycle.

The FDA allows us to validate the methodology used to compute the IDA: The joint torques obtained via the IDA are dynamically consistent with the motion, and those torques are the actual ones because when they are used to compute an FDA they provide a motion extremely close to the captured one (without adding neither residual torques nor a controller).

The instability of the system after the $90 \%$ of the cycle can be related to numerical inaccuracies and to errors in the integration of the motion equations along time. In order to improve the results for the whole captured motion, a simple PD controller has been implemented and its dynamic effect is shown to be really low, providing a way to easily control the biomechanical system during the forward dynamics simulations.

The IDA looks at each discretized instant of time separately and the FDA implies the dynamically consistent solution of the motion equations over the full recorded period. This study shows that if the IDA is computed using a dynamically consistent method, the results obtained via the IDA can be used to compute a FDA obtaining good results along almost the full gait cycle.

This simple method has been implemented to get experience in the field of forward dynamics simulation and it is a first step of a future work in human gait prediction.

\section{ACKNOWLEDGMENTS}

This work is supported by the Spanish Ministry of Science and Innovation under the project DPI2009-13438-C03. The support is gratefully acknowledged.

\section{REFERENCES}

[1] Ambrósio, J. A. C., Kecskeméthy A., 2007, “Multibody Dynamics of Biomechanical Models for Human Motion via Optimization", Computational Methods in Applied Sciences, 4, pp. 245-272.

[2] Ackermann, M., Van den Bogert, A. J., 2010, “Optimality Priciples for Model-Based Prediction of Human Gait”, J. Biomechanics, 43, pp. 1055-1060. 
[3] Thelen, D. G., Anderson, F. C., 2006, “Using Computed Muscle Control to Generate Forward Dynamic Simulations of Human Walking from Experimental Data", J. Biomechanics, 39(6), pp. 1107-1115.

[4] Van den Bogert, A. J., Blana D., Heinrich D., 2011, "Implicit Methods for Efficient Musculoskeletal Simulation and Optimal Control”, Symp. Human Body Dynamics, Procedia IUTAM, 2, pp. 297-316.

[5] Xiang, Y., Arora, J. S., Rahmatalla, S. and Abdel-Malek, K. 2009, "Optimization-Based Dynamic Human Walking Prediction”, Int. J. Numerical Methods in Engineering, 79 (6), pp. 667-695.

[6] Fregly, B. J., Reinbolt, J. A., Rooney, K. L., Mitchell, K. H., Chmielewski, T. L., 2007, "Design of Patient-Specific Gait Modifications for Knee Osteoarthritis Rehabilitation”, IEEE Trans. Biomedical Engineering, 54(9), pp. 1687-1695.

[7] Kim, J. H., Abdel-Malek, K., Yang, J., Marler, R. T., 2006, "Prediction and Analysis of Human Motion Dynamics Performing Various Tasks”, Int. J. Human Factors Modelling and Simulation, 1(1), pp. 69-94.

[8] Cuadrado, J., Pàmies-Vilà, R., Lugrís, U., Alonso F.J., 2011, "A Force-Based Approach for Joint Efforts Estimation during the Double Support Phase of Gait", Symp. Human Body Dynamics, Procedia IUTAM, 2, pp. 26-34.

[9] Vaughan, C.L., 1982, "Smoothing and differentiation of displacement-time data: an application of splines and digital filtering", International Journal of Bio-Medical Computing, 13, pp. 375-386.

[10] Chao, G. A., Rim, L. S., 1973, "Application of optimization principles in determining the applied moments in human leg joints during gait”. Journal of Biomechanics, 6, pp. 497-510.

[11] Cahouët, V., Luc, M., Amarantini, D., 2002, "Static optimal estimation of joint accelerations for inverse dynamics problem solution", Journal of Biomechanics, 35, pp. 1507-1513.

[12] Remy, C. D., Thelen D. G., 2009 "Optimal estimation of dynamically consistent kinematics and kinetics for forward dynamic simulation of gait”, Journal of Biomechanical Engineering, 131 (3), pp. 031005.

[13] Kuo, A. D., 1998, “A least squares estimation approach to improving the precision of inverse dynamics computations”, J. Biomechanical Engineering, 120, pp. 148-159.

[14] Ackermann, M., Schiehlen, W., 2006, "Dynamic Analysis of Human Gait Disorder and Metabolical Cost Estimation”, Arch. Applied Mechanics, 75, pp. 569-594.

[15] Ambrósio, J. A. C., Silva, M., Abrantes, J., 1999, "Inverse Dynamic Analysis of Human Gait Using Consistent Data”, Proc. Int. Symp. Computer Methods in Biomechanics and Biomedical Engineering, Lisboa, Portugal.
[16] Dumas, R., Nicol, E., Chèze, L., 2007, "Influence of the 3D Inverse Dynamic Method on the Joint Forces and Moments During Gait”, J. Biomechanical Engineering, 129(5), pp. 786-790.

[17] Alonso, F.J., Cuadrado, J., Lugrís, U., Pintado, P., 2010, "A Compact Smoothing-Differentiation and Projection Approach for the Kinematic Data Consistency of Biomechanical Systems, Multibody System Dynamics, 24(1), pp. 67-80.

[18] García de Jalón, J., Bayo, E., 1994, "Kinematic and Dynamic Simulation of Multibody Systems: the RealTime Challenge”, Springer-Verlag, New York.

[19] Pàmies-Vilà, R., 2012, “Application of Multibody Dynamics Techniques to the Analysis of Human Gait”, PhD Thesis, Universitat Politècnica de Catalunya, Spain.

[20] Davis, B. L., Cavanagh, P. R., 1993, "Decomposition of superimposed ground reaction forces into left and right force profiles”. J. Biomechanics, 26, pp. 593-597.

[21] Ren, L., Jones, R. K., Howard, D., 2008, "Whole body inverse dynamics over a complete gait cycle based only on measured kinematics” J. Biomechanics, 41, pp. 27502759.

[22] Cappozzo, A., Tommaso, L., Pedotti A., 1975, “A General Computing Method for the Analysis of human Locomotion”, J. Biomechanics, 8, pp. 307-320.

[23] Forner-Cordero A., Koopman H.J.F.M., Van der Helm F. C. T., 2006, "Inverse Dynamics Calculations during Gait with Restricted Ground Reaction Force Information from Pressure Insoles”, Gait and Posture, 23(2), pp. 189-199.

[24] McCaw, S. T., De Vita, P., 1995, "Errors in Alignment of Centre of Pressure and Foot Coordinates Affect Predicted Lower Extremity Torques”, J. Biomechanics, 28(8), pp. 985-988.

[25] Winter, D. A., 1991, "The Biomechanics and Motor Control of Human Gait: Normal, Elderly and Pathological”, University of Waterloo Press, Waterloo, Canada.

[26] Astrom, K. J., Hagglund, T, 1995, "PID controllers:theory, design, and tuning” Instrument Society of America, Research Triangle Park, North Carolina. 